\title{
Deprescribing Inappropriate Proton Pump Inhibitors in a Family Medicine Residency Practice Office
}

\author{
Andrew Lai, DO | Amy Odom, DO | Steven E. Roskos, MD | Julie P. Phillips, MD, MPH
}

PRiMER. 2021;5:43.

Published: 11/2/2021 | DOI: 10.22454/PRiMER.2021.290175

\section{Abstract}

Introduction: Proton pump inhibitors (PPIs) are often prescribed beyond their medically-approved duration of use. Long-term PPI use has been linked with numerous adverse effects and contributes to polypharmacy. This study sought to understand the effect of evidence-based education to resident and faculty physicians on deprescribing inappropriate PPIs. We hypothesized that this educational intervention would result in fewer inappropriate PPI prescriptions.

Methods: We performed a chart review on all adult patients in a family medicine residency program practice, identifying those inappropriately taking PPIs. All physicians in the practice participated in an educational intervention regarding deprescribing inappropriate PPIs and were given frequent reminders to continue their deprescribing efforts. We reviewed charts at the end of the study to identify patients with successful deprescribing attempts.

Results: Of the 187 patients prescribed inappropriate PPIs in the study, 100 remained on PPIs at the end of the study ( $46.6 \%$ success rate). There was a significant decrease in the number of patients inappropriately prescribed PPIs by each physician over the course of the study, from a mean of 17.0 (beginning) to 9.1 (end).

Conclusion: Physician education with reminders is an effective means of reducing the number of inappropriate PPIs prescribed in a family medicine residency practice. Future studies could investigate the effectiveness of educational interventions with other medication classes that are often prescribed inappropriately.

\section{Introduction}

Since their introduction to the market in the 1980s, proton pump inhibitors (PPIs) have often been the first-line medical therapy for patients in the treatment of their gastroesophageal reflux disease (GERD). Given the drug class' powerful ability to suppress stomach acid production, both the United States Food and Drug Administration and the American Gastroenterological Association recommend a limited duration of use (4 to 8 weeks) in the treatment of GERD with a PPI. ${ }^{1}$ Despite this recommendation, many patients remain on PPIs well beyond their intended duration of use. 
The medical literature has cited numerous associations linking the use of long-term PPIs to adverse effects in patients. The increased $\mathrm{pH}$ environment of the gastrointestinal tract caused by the use of PPIs has been linked with reduced vitamin and mineral absorption, such as vitamin $\mathrm{B}_{12}$, calcium (leading to osteoporosis and subsequent bone fractures), iron and magnesium. ${ }^{2-5}$ The increased $\mathrm{pH}$ environment also weakens the innate immune system, which has been associated with increased risk of Clostridium difficile colitis, aspiration and community-acquired pneumonia, small intestinal bacterial overgrowth, and spontaneous bacterial peritonitis in patients with liver cirrhosis. ${ }^{6-9}$ Pharmacokinetics and pharmacodynamics are also altered by the increased $\mathrm{pH}$, leading to reports of diminished activity of clopidogrel and decreased absorption of certain medications, such as ketoconazole. ${ }^{10,11}$ Other associations noted in the literature with long-term PPI use, albeit due to unknown mechanisms, include acute kidney injury, chronic kidney disease and dementia. ${ }^{12-14}$ Additionally, inappropriate PPI prescriptions contribute to polypharmacy, particularly in our increasingly aging society. ${ }^{15}$ Polypharmacy, defined as regular use of at least five medications, increases the risk of adverse consequences and increases the patient's financial burden. ${ }^{16}$ Thus, every effort should be made by family physicians to deprescribe inappropriate PPIs. Deprescribing is defined as the act of discontinuing one or more medications that are either potentially harmful or no longer required, through a medically supervised, patient-centered process. ${ }^{17}$

The medical literature addressing how to deprescribe inappropriate PPIs is vast. Many approaches have been found effective, including the use of evidence-based decision aids; patient-centered, shared decision-making; frequent reminders to physicians and patients; and use of electronic medical records. ${ }^{18-20}$ Deprescribing PPIs has also been described in the literature in residency clinic settings, in the form of quality improvement projects. ${ }^{21,22}$ Little research, however, has been done on the impact of educating resident physicians on the topic of deprescribing PPIs.

This study sought to understand the effect of providing evidence-based education to physicians on deprescribing inappropriate PPIs. We hypothesized that this educational intervention would improve prescribing practices and result in fewer inappropriate PPI prescriptions.

\section{Methods}

The Sparrow Hospital Institutional Review Board exempted this study from review. Using tools available in the electronic medical record, we performed a chart review on all adult patients in a family medicine residency office who were prescribed PPIs. We defined inappropriately prescribed PPIs as prescriptions lacking a clear medical diagnosis supporting prolonged use (eg, Barrett's esophagus, gastrointestinal bleeding prophylaxis with concurrent high-dose NSAID use, history of upper gastrointestinal bleeding, severe-grade esophagitis, Zollinger-Ellison syndrome).

The principal investigator gave physicians in the office lists of their patients who were found to have inappropriately prescribed PPIs on their medication lists. All physicians who agreed to participate in the study also attended an educational, 1-hour PowerPoint lecture during a scheduled residency didactics session, led by the principal investigator, on the topic of deprescribing inappropriate PPIs. The presentation detailed inappropriate PPI usage and offered strategies for how to deprescribe PPIs (eg, gradual tapering off medication, concomitant use of $\mathrm{H}_{2}$ receptor antagonists, nonpharmacologic approaches to symptom management).

Over a 4-month period, physicians in the study were routinely reminded of their deprescription efforts through messages sent to physicians via the electronic medical record and face-to-face conversations in the clinic by the prinicpal investigator. The principal investigator monitored participating physicians' efforts throughout the study period through use of the electronic medical record, assessing whether patients still had their inappropriate PPIs on their medication lists or if their dosage/frequency of use had decreased. 
We used one-way repeated measures (ANOVA) to compare the number of patients inappropriately prescribed PPIs at the beginning, middle, and end of the study. We excluded patients who left the practice, were transitioned to hospice care, or died during the study.

\section{Results}

Two hundred patients met inclusion criteria (PPIs on their medication list without a medical diagnosis to support prolonged use). Of these 200 , we excluded 13 due to leaving the practice, transitioning to hospice care, or dying ( $n=187$ ). By the middle of the study, (day 66), 130 patients remained on inappropriate PPIs, and by the end of the study (day 124), 100 patients remained (46.6\% success rate). All 11 senior resident and attending physicians in the office participated. There was a significant decrease in the number of patients inappropriately prescribed PPIs by each participating physician over the course of the study, from a mean of 17.0 at the beginning of the study, to 11.8 midway through the study, and 9.1 at the end of the study (Figure 1). This decrease was statistically significant, and the effect size was large $\left(P<.001\right.$, partial $\left.\eta^{2}=0.928\right)$.

\section{Discussion}

Results suggest that physician education with reminders is an effective means of reducing the number of inappropriate PPIs prescribed in a family medicine resident practice. Educational interventions may be a helpful tool in promoting deprescribing efforts alongside other deprescribing methods noted in the literature, particularly among resident physicians.

This study took place in a single residency clinic, limiting its sample size of physicians and patients. The study's principal investigator was a resident in the clinic, which may have increased residents' engagement. No pediatric patients were included; inclusion of pediatric patients in future investigations could lead to broader applicability of findings. Further studies could investigate the use of similar interventions on other medication classes commonly prescribed inappropriately, such as bisphosphonates and statins.

\section{Conclusions}

We demonstrated a favorable improvement in inappropriate PPI deprescribing and prescriber knowledge through focused education and feedback. Further use of educationally-driven deprescribing interventions may prove beneficial in encouraging cost-effective, evidence-based, patient-centered care.

\section{Tables and Figures}


Figure 1: Mean Number of Patients Inappropriately Prescribed PPIs by Each Physician $(n=11)$ Over the Course of the Study (Error Bars Represent Standard Deviations)

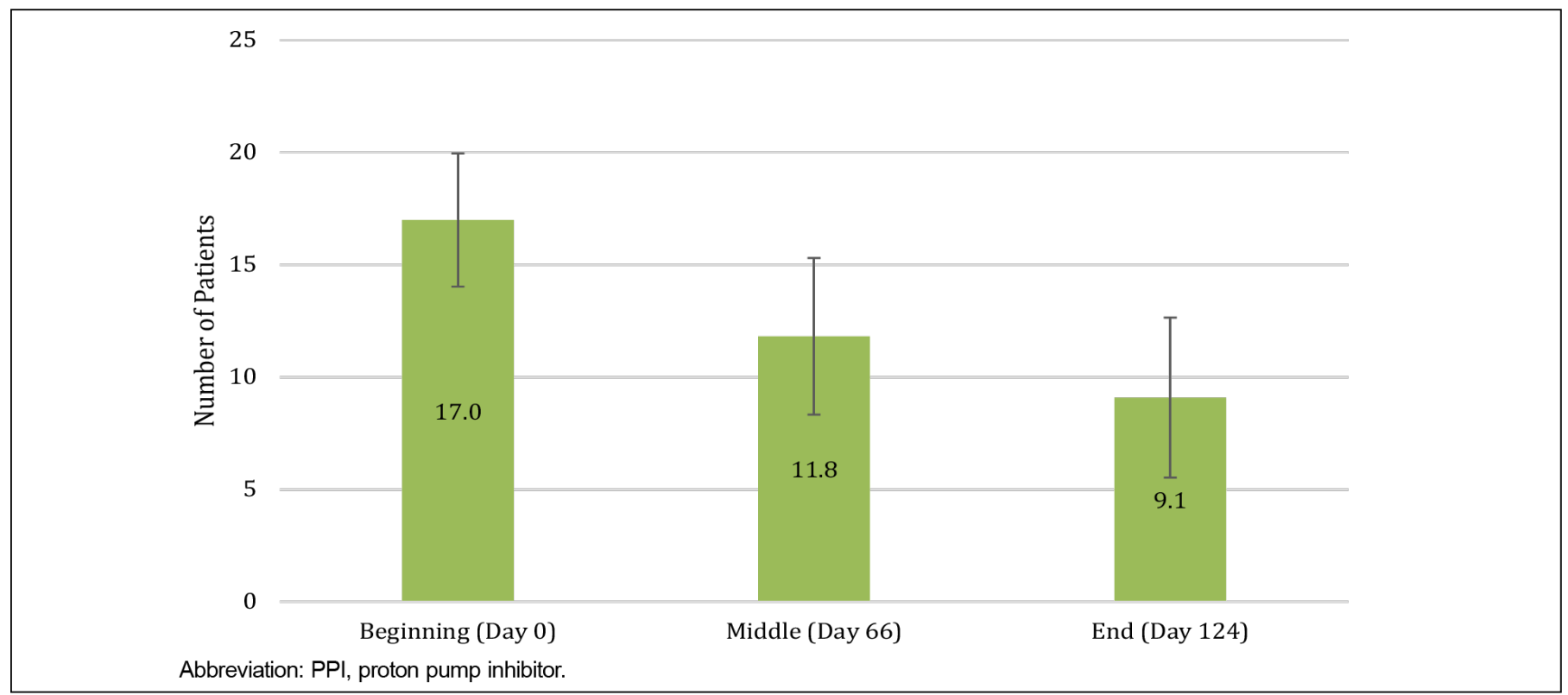

\section{Acknowledgments}

The authors thank the doctors of Sparrow Medical Group Mason Family Medicine Residency practice for participating in the study.

Presentations: This paper was presented at the following conferences:

- American College of Osteopathic Family Physicians 2021, Poster Presentation Session, March 18, 2021 (Virtual)

- Mid-Michigan Regional Research Day, April 22, 2021 (Virtual)

- Michigan Osteopathic Association Spring 2021 Virtual Research Exhibit, May 1, 2021 (Virtual)

- Society of Teachers of Family Medicine 2021 Annual Spring Conference, May 3, 2021 (Virtual)

\section{Corresponding Author}

Andrew Lai, DO

18-16945 Leslie Street, Newmarket, ON, Canada L3Y9A2. 905-868-8689.

andrew.m.lai@gmail.com

\section{Author Affiliations}

Andrew Lai, DO - Sparrow Hospital-Michigan State University Family Medicine Residency Program, Lansing, MI Amy Odom, DO - Sparrow Hospital-Michigan State University Family Medicine Residency Program, Lansing, MI Steven E. Roskos, MD - Michigan State University College of Human Medicine and Sparrow Hospital-Michigan State University Family Medicine Residency Program, Lansing, MI

Julie P. Phillips, MD, MPH - Michigan State University College of Human Medicine and Sparrow HospitalMichigan State University Family Medicine Residency Program, Lansing, MI

\section{References}

1. Katz PO, Gerson LB, Vela MF. Guidelines for the diagnosis and management of gastroesophageal reflux disease. Am J Gastroenterol. 2013;108(3):308-328. doi:10.1038/ajg.2012.444 
2. Lam JR, Schneider JL, Zhao W, Corley DA. Proton pump inhibitor and histamine 2 receptor antagonist use and vitamin B12 deficiency. JAMA. 2013;310(22):2435-2442. doi:10.1001/jama.2013.280490

3. Zhou B, Huang Y, Li H, Sun W, Liu J. Proton-pump inhibitors and risk of fractures: an update metaanalysis. Osteoporos Int. 2016;27(1):339-347. doi:10.1007/s00198-015-3365-X

4. Lam JR, Schneider JL, Quesenberry CP, Corley DA. Proton pump inhibitor and histamine-2 receptor antagonist use and iron deficiency. Gastroenterology. 2017;152(4):821-829.e1. doi:10.1053/j.gastro.2016.11.023

5. Cheungpasitporn W, Thongprayoon C, Kittanamongkolchai W, et al. Proton pump inhibitors linked to hypomagnesemia: a systematic review and meta-analysis of observational studies. Ren Fail. 2015;37(7):1237-1241. doi:10.3109/0886022X.2015.1057800

6. Kwok CS, Arthur AK, Anibueze Cl, Singh S, Cavallazzi R, Loke YK. Risk of Clostridium difficile infection with acid suppressing drugs and antibiotics: meta-analysis. Am J Gastroenterol. 2012;107(7):1011-1019. doi:10.1038/ajg.2012.108

7. Johnstone J, Nerenberg K, Loeb M. Meta-analysis: proton pump inhibitor use and the risk of communityacquired pneumonia. Aliment Pharmacol Ther. 2010;31(11):1165-1177. doi:10.1111/j.1365-2036.2010.04284.x

8. Su T, Lai S, Lee A, He X, Chen S. Meta-analysis: proton pump inhibitors moderately increase the risk of small intestinal bacterial overgrowth. J Gastroenterol. 2018;53(1):27-36. doi:10.1007/s00535-017-1371-9

9. Trikudanathan G, Israel J, Cappa J, O'Sullivan DM. Association between proton pump inhibitors and spontaneous bacterial peritonitis in cirrhotic patients - a systematic review and meta-analysis. Int J Clin Pract. 2011;65(6):674-678. doi:10.1111/j.1742-1241.2011.02650.x

10. Luinstra $M$, Naunton $M$, Peterson GM, Bereznicki L. PPI use in patients commenced on clopidogrel: a retrospective cross-sectional evaluation. J Clin Pharm Ther. 2010;35(2):213-217. doi:10.1111/j.1365-2710.2009.01089.x

11. Ogawa R, Echizen H. Drug-drug interaction profiles of proton pump inhibitors. Clin Pharmacokinet. 2010;49(8):509-533. doi:10.2165/11531320-000000000-00000

12. Chen G, Ning LJ, Qin Y, Zhao B, Mei D, Li XM. Acute kidney injury following the use of different proton pump inhibitor regimens: A real-world analysis of post-marketing surveillance data. J Gastroenterol Hepatol. 2021;36(1):156-162. doi:10.1111/jgh.15151

13. Lazarus $B$, Chen $Y$, Wilson FP, et al. Proton pump inhibitor use and the risk of chronic kidney disease. JAMA Intern Med. 2016;176(2):238-246. doi:10.1001/jamainternmed.2015.7193

14. Gomm W, von Holt K, Thomé F, et al. Association of proton pump inhibitors with risk of dementia: a pharmacoepidemiological claims data analysis. JAMA Neurol. 2016;73(4):410-416. doi:10.1001/jamaneurol.2015.4791

15. Zed PJ. Deprescribing proton pump inhibitors. Can J Hosp Pharm. 2018;71(5):291-292.

16. Halli-Tierney AD, Scarbrough C, Carroll D. Polypharmacy: evaluating risks of and deprescribing. Am Fam Physician. 2019;100(1):32-38.

17. Page A, Clifford R, Potter $K$, Etherton-Beer $C$. A concept analysis of deprescribing medications in older people. J Pharm Pract Res. 2018;48(2):132-148. doi:10.1002/jppr.1361

18. Reeve E, Andrews JM, Wiese MD, Hendrix I, Roberts MS, Shakib S. Feasibility of a patient-centered deprescribing process to reduce inappropriate use of proton pump inhibitors. Ann Pharmacother. 2015;49(1):29-38. doi:10.1177/1060028014558290

19. Odenthal DR, Philbrick AM, Harris IM. Successful deprescribing of unnecessary proton pump inhibitors in a primary care clinic. J Am Pharm Assoc (Wash DC). 2020;60(1):100-104.

doi:10.1016/j.japh.2019.08.012

20. Walsh K, Kwan D, Marr P, Papoushek C, Lyon WK. Deprescribing in a family health team: a study of chronic proton pump inhibitor use. J Prim Health Care. 2016;8(2):164-171. doi:10.1071/HC15946

21. Boster J, Lowry LE, Bezzant ML, Kuiper B, Surry L. Reducing the inappropriate use of proton pump 
inhibitors in an internal medicine residency clinic. Cureus. 2020;12(1):e6609. doi:10.7759/cureus.6609

22. Nallapeta N, Reynolds JL, Bakhai S. Deprescribing proton pump inhibitors in an academic, primary care clinic: quality improvement project. J Clin Gastroenterol. 2020;54(10):864-870.

doi:10.1097/MCG.0000000000001317

Copyright $(2021$ by the Society of Teachers of Family Medicine 